\title{
Analysis of Time of Day Fare Discounts on Urban Mass Transit Travel Behavior, Crowding, and Waiting Time
}

\author{
Xiao Guo and Huijun Sun \\ MOE Key Laboratory for Urban Transportation Complex Systems Theory and Technology, \\ Beijing Jiaotong University, Beijing 100044, China \\ Correspondence should be addressed to Huijun Sun; hjsun1@bjtu.edu.cn
}

Received 20 February 2014; Revised 18 July 2014; Accepted 18 July 2014; Published 12 August 2014

Academic Editor: Hu Shao

Copyright (C) 2014 X. Guo and H. Sun. This is an open access article distributed under the Creative Commons Attribution License, which permits unrestricted use, distribution, and reproduction in any medium, provided the original work is properly cited.

Every morning, commuters select the regularly dispatched urban mass transit for traveling from a residential area to a workplace. This paper aims to find an optimal discount fare and time intervals on morning peak hour. As a direct and flexible traffic economic instrument, fares can influence commuters' behavior. Therefore, fare discount has been proposed to regulate traffic flow in different time. Two models have been analyzed to describe it with schedule delay because of the travel demand size. The first objective function is constructed on pressure equalization when the travel demand is small. The other objective function is to minimize total waiting time when the travel demand is large. In the end, numerical examples based on an artificial network are performed to characterize fare discount models.

\section{Introduction}

Problems of sustainability in transportation are already quite noticeable in almost all large cities. Due to congestion, more waiting time is wasted on the roads, and commuters are more likely to get stressed and frustrated, which in turn causes accidents. Forecasts of traffic condition are less reliable, so punctual arrival becomes difficult and travelers suffer losses from early departure or late arrival [1]. As an integral part and essential feature of cities, public transportation plays an important role in developed and developing countries. Providing efficient public transportation systems has been recognized as a potential way of alleviating traffic congestion, improving mobility, mitigating air pollution, and reducing energy consumption. It also provides a convenient way to connect residential areas and CBD [2].

The crowding on the platform has been generated on rush hours. Commuters usually decide the departure time to minimize the individual travel cost. In early literature, the studies about public transit were based on the traditional marginal cost pricing theory [3]. Mohring [4, 5] developed a microeconomic foundation for public transportation services with fixed demand, which was quite useful for long-term public transit service planning in a static sense. However, this model cannot describe a commuter's time-of-use behavior on rush hours.

Some studies consider the time dimension to describe a commuter's behavior. Various time-varying pricing models and algorithms have been proposed to determine the tolls for system performance optimization under given physical and economic pricing constraints. This first work is done by Vickrey [6] who develops a dynamic toll model with deterministic queuing theory, which leads to an equilibrium of costs on all commuters. Subsequently, this model has been extended by many others $[7,8]$, and the concept of schedule delay cost has been widely used in proposing various commuting equilibrium models [9-13]. In 1982, Small [14] accomplished an important empirical study and calibrated the disutility function of schedule delay using the survey data in the United States.

In a transit system, it is generally unpractical to provide the capacity for all commuters to arrive on a train during peak hours in congested cities; so some of commuters may have to wait until a train arrives that they can board. They probably have to stand during the peak period and suffer discomfort due to congestion on platform. Kraus and Yoshida [15] and Kraus [16] analyze the economic analysis of commuters' time-of-use decision and examine the optimal pricing and 
service in an urban mass transit system. Huang et al. [17, 18] introduce an in-vehicle congestion function in modeling the choice of urban mass transit runs. Li and Hensher [19] review public transport crowding valuation research. In those papers, a commuter's cost contains the cost of waiting time at the origin transit stop and is similar to the cost of queuing time in the bottleneck model.

Some papers talk about the transit frequency when they consider waiting time on rush hours $[20,21]$. Yu et al. [21] define that it is constant. Thus, they cannot describe the changing of commuters' waiting time. In this paper, waiting time is redefined to describe the congestion phenomenon. Waiting time is different from that as we discussed above. It is a measure of a scalar congestion. Meanwhile, it is also different from the waiting time in traditional bottleneck model. In bottleneck model, waiting time has relationship to departure rate, and it is different due to queue length. In our model, waiting time is not only related to the departure time, but also related to the congestion in a train. According to our assumption, this concept can explain the reason of congestion through our model.

On the other side, transit service level is low in most cities of China. Some passengers have to stay on platform, and there exist seats on off-peak hours. Fare can influence commuters' behavior as a direct and flexible traffic economic instrument. Therefore, this paper proposes fare discount model to enhance the transit service level. Time-varying transit fares have been investigated to solve congestion when timetable is fixed. Low fare would attract some commuters who are in the peak hour transfer to the lower price period. They will change some commuters' behavior and alleviate the congestion in public transit system.

This paper investigates time-varying railroad fares discount models. There exist two kinds of models because of travel demand. The small travel demand is named when the max number of passengers in a train is smaller than the capacity. According to assumptions, the waiting time is a fixed value to everyone. Thus, the objective function is constructed on pressure equalization. It is the total sum of squared differences between the ridership and the number of seats in trains. The second situation is discussed in the condition of large travel demand. The objective function is to minimize total waiting time as described in former. This paper explores the consequences of fare adjustment in the urban railroad mode. The results show that the structure of the ticket fare discount does indeed influence the behavior of the commuters.

The paper is organized as follows. Section 2 introduces generalized travel cost for urban railroad with the schedule delay penalty. In Section 3, two different models have been considered to alliviate the congstion. In Section 4, numerical analyses are provided to illustrate the models. Section 5 concludes the paper.

\section{Basic Assumptions and Generalized Travel Cost Function}

In this paper, a railroad connecting the residents to the CBD has been considered. In the morning, there are $N$ commuters who have to make their trips to the CBD for work by this public transit. Due to the physical constraint of the train's capacity, most commuters necessarily arrive earlier or later than the work starting time, and some commuters have to stay on platform waiting for the following trains. In such a case, commuters have to incur the costs of schedule delay and the costs of waiting time. Some assumptions have been described in the next section before generalized travel cost fuctions are displayed.

2.1. Basic Assumptions. To facilitate the presentation of the essential ideas of this paper, all commuters are assumed to be identical in perceiving the time value and schedule delay penalty. The following basic assumptions are made in this paper.

(1) The commuters who are in a train have the same travel cost. That is to say, those commuters are assumed to have the same waiting time when they are on the same train.

(2) Commuters must obey the first-come-first-served basis. All commuters can board train when the number of commuters is smaller than the capacity of the train. Only the former $s$ ( $s$ represents the capacity of a train.) commuters can board train when the number of commuters on platform is bigger than $s$. The rest of them need to wait the following trains.

2.2. Generalized Travel Cost Function. This paper aims to provide an explaination of how fare discounts affect cummuters' travel behavior on rush hours. The cost experienced by a railroad commuter incldues the in-vehicle time, the waiting time on platform, the schedule delay of arrival at workplace, the urban railroad fare, and the body discomfort. In this paper, the waiting time has been redefined to describe the congestion phenomenon on the platform. The schedule delay is assumed to be fixed in each train when the timetable is fixed. The congestion discomfort is generated by body congestion in the train carriage, which is a monotonically increasing function of the ridership.

Travel Time. Firstly, let $[0, A]$ be the morning peak operating time interval. According to each urban railroad's departing time, $[0, A]$ can be divided into $n$ subintervals $\left(a_{i-1}, a_{i}\right]$, where $a_{0}=0, a_{n}=A$, and $i \in\{1,2, \ldots, n\} . a_{i}$ is the departing time of the $i$ th urban railroad. Let $w(t)$ be the waiting time when a commuter arrives at platform at time $t$, and the departing time of commuters in the same vehicle obeys the uniform distribution. $[0, A]$ can be divided into $n$ subintervals $\left(t_{i-1}, t_{i}\right]$, where $t_{0}=0, t_{n}=A$, and $i \in\{1,2, \ldots, n\}$. $t_{i-1}$ is the time of a commuter who arrives at platform and boards a vehicle as the first passenger. $t_{i}$ is the time of a commuter who arrives at platform and boards the vehicle as the last passenger. According to the former assumption, the waiting time of commuters in the same vehicle can be easily calculated $w_{i}=$ $a_{i}-\left(t_{i-1}+t_{i}\right) / 2, i \in\{1,2, \ldots, n\}$. Constraint $t_{i} \leq a_{i}, i \in$ $\{1,2, \ldots, n\}$, can ensure that no one can get on vehicle after 
the vehicle's leaving platform. Let $\eta_{1}$ be the unit cost of travel time. Then the travel time costs are $\eta_{1}\left(T+w_{i}\right), i \in\{1,2, \ldots, n\}$.

Schedule Delay. Schedule delay means the time with respect to the work starting time (assumed to be same for all commuters in the same vehicle). Let $t^{*}$ be the work starting time; if a commuter arrives at workplace before $t^{*}$, the schedule delay is $t^{*}-\left(a_{i}+T\right)$; if a commuter arrives at workplace on $t^{*}$, then $t^{*}-\left(a^{*}+T\right)=0$, where $a^{*}=t^{*}-T$ is the perfect departure time; if a commuter arrives at workplace after $t^{*}$, the schedule delay is $\left(a_{i}+T\right)-t^{*}$. Let $\eta_{2}$ be the unit cost of schedule delay time-early and let $\eta_{3}$ be the unit cost of schedule delay timelate. An early schedule delay is $\eta_{2}\left[t^{*}-\left(a_{i}+T\right)\right]$, and a late schedule delay is $\eta_{3}\left[\left(a_{i}+T\right)-t^{*}\right]$.

Congestion Effect. The congestion effect is generated by body congestion in urban railroad carriage. $g\left(x_{i}\right)$ represents the body congestion cost of unit in-carriage time which is a monotonically increasing function of the number of commuter $x_{i}$ in the urban railroad [17]. Let $g\left(x_{i}\right)=G\left(x_{i} / x_{0}\right)$, where $G$ represents a parameter $(G>0)$ and $x_{i}$ denotes the number of commuters in a urban railroad depart at time $a_{i}$, $i=\{1, \ldots, n\} . x_{0}$ represents the number of seats.

The Urban Railroad Fare. The urban railroad fare is represented by $p$.

Integrating the travel time, schedule delay, travel comfort, and travel fare, a commuter's generalized travel cost can be formulated as follows:

$$
C(t)=\left\{\begin{array}{cr}
\eta_{1}\left(T+a_{i}-\frac{t_{i-1}+t_{i}}{2}\right)+\eta_{2}\left[t^{*}-\left(a_{i}+T\right)\right] \\
+G \frac{x_{i}}{x_{0}}+p & \text { if } a_{i}+T \leq t^{*} \\
\eta_{1}\left(T+a_{i}-\frac{t_{i-1}+t_{i}}{2}\right)+\eta_{3}\left[\left(a_{i}+T\right)-t^{*}\right] \\
+G \frac{x_{i}}{x_{0}}+p & \text { if } a_{i}+T \geq t^{*} .
\end{array}\right.
$$

\section{The Schedule Delay Penalty Models}

This section describes two formulations for the problem of finding a user-equilibrium strategy assignment. The first model describes the schedule delay penalty model when the travel demand is small. In this model, some factors can be determined by assumptions.

3.1. The Fare Discount Model under Equal Pressure. In this section, the small travel demand has been considered in this schedule delay penalty model, in which the ridership in each train is smaller than $s$. This means all commuters waiting on platform can get on the vehicle. The case can be described as $t_{i}=a_{i}, i=1, \ldots, n-1$. So the waiting time can be caculated accurately $w(t)=\gamma \cdot \Delta t$, where $\gamma$ represents the ratio of average waiting time and $\Delta t$ represents headway. $\gamma$ is approximately $1 / 2$ if commuter arrivals at station follow a uniform distribution, while vehicles arrive at station deterministically. The travel time cost is $\eta_{1}(T+$ $(1 / 2) \Delta t)$. The times of commuters from the residential area to the nearby station and the end urban railroad to the workplace are fixed. Thses two values do not affect the calculation results. Both of them can be calculated into $T$.

The aim is to alleviate traffic congestion through reducing the ridership in peak hour and shifting commuters from the peak to the off-peak period. When the edges' fare is smaller than the peak hour's, some commuters will be attracted to change their departure time and transfer to the edges. This paper hopes to calculate the discount intervals and the size of the fare discount under equal pressure. This process can be described as follows.

We set the discount time intervals $\left[a_{0}, a_{k}\right]$ and $\left[a_{l}, a_{n}\right]$, where $a_{k} \leq t^{*}-T, a_{l} \geq t^{*}-T$. Let the discounted railroad fare be $p \theta$, where $0 \leq \theta \leq 1$. The discounted intervals and discounted railroad fare would be calculated to minimize the variance between the ridership and the number of seats in a train.

According to the assumptions, the travel cost can be divided into four classes. The first class is before $t^{*}$, and the travel cost contains fare discount; the second class is before $t^{*}$, and the travel cost contains fare discount; the third class is after $t^{*}$, and the travel cost contains fare discount; the last class is after $t^{*}$, and the travel cost contains fare discount.

The travel cost of the first class could be described by the following formula:

$$
\begin{aligned}
C_{i}(t)= & \eta_{1}\left(T+\frac{1}{2} \Delta t\right)+\eta_{2}\left[t^{*}-\left(a_{i}+T\right)\right] \\
& +G \frac{x_{i}}{x_{0}}+p \theta, \quad t \in\left[a_{0}, a_{k}\right] .
\end{aligned}
$$

The travel cost of the second class could be described by the following formula:

$$
\begin{aligned}
C_{i}(t)= & \eta_{1}\left(T+\frac{1}{2} \Delta t\right)+\eta_{2}\left[t^{*}-\left(a_{i}+T\right)\right] \\
& +G \frac{x_{i}}{x_{0}}+p, \quad t \in\left(a_{k}, a^{*}\right] .
\end{aligned}
$$

The travel cost of the third class could be described by the following formula:

$$
\begin{aligned}
C_{i}(t)= & \eta_{1}\left(T+\frac{1}{2} \Delta t\right)+\eta_{3}\left[\left(a_{i}+T\right)-t^{*}\right] \\
& +G \frac{x_{i}}{x_{0}}+p, \quad t \in\left(a^{*}, a_{l}\right] .
\end{aligned}
$$

The travel cost of the last class could be described by the following formula:

$$
\begin{aligned}
C_{i}(t)= & \eta_{1}\left(T+\frac{1}{2} \Delta t\right)+\eta_{3}\left[\left(a_{i}+T\right)-t^{*}\right] \\
& +G \frac{x_{i}}{x_{0}}+p \theta, \quad t \in\left(a_{l}, a_{n}\right] .
\end{aligned}
$$

All the commuters have been divided into four classes. At equilibrium state, all commuters incur the same travel cost 
no matter what time they leave home. This model can be described as the following programming problem:

$$
\min \left(\sum_{i=1}^{n}\left(x_{i}-x_{0}\right)^{2}\right)
$$

s.t. $(2)-(5)$

$$
\begin{gathered}
\sum_{1}^{n} x_{i}=N . \\
C_{1}(t)=C_{2}(t)=\cdots=C_{n}(t) .
\end{gathered}
$$

The objective function (6) means to minimize the total sum of squared differences between the ridership and the number of seats in trains. Constraints (2)-(5) state the travel cost in different trains. Constraint (7) represents the flow conservation. Constraint (8) guarantees the travel costs of each commuter are equal at equilibrium.

\subsection{The Fare Discount Model to Minimize the Total Waiting} Time. In this section, the railroad fare discount has been considered when the travel demand is large. Like the former, the discount intervals and railroad fare have been analyzed. Given intervals, we set the discount intervals $\left[a_{0}, a_{k}\right]$ and $\left[a_{l}, a_{n}\right]$, where $a_{k}<t^{*}-T, a_{l}>t^{*}-T$, and $a_{k}<a_{l}$. Let the discount ticket fare be $p \theta$, where $0 \leq \theta \leq 1$. This paper hopes to calculate the discount intervals and the size of the fare discount to minimizing the total waiting time. According to the assumptions, the travel cost can be divideed into four classes. The first class is before $t^{*}$, the travel cost with discount; the second class is before $t^{*}$, the travel cost without discount; the third class is after $t^{*}$, the travel cost without discount; the last class is after $t^{*}$, the travel cost with discount.

The travel cost of the first class could be described by the following formula:

$$
\begin{aligned}
C_{i}(t)= & \eta_{1}\left(T+a_{i}-\frac{t_{i-1}+t_{i}}{2}\right)+\eta_{2}\left[t^{*}-\left(a_{i}+T\right)\right] \\
& +G \frac{x_{i}}{x_{0}}+p \theta, \quad t \in\left[a_{0}, a_{k}\right] .
\end{aligned}
$$

The travel cost of the second class could be described by the following formula:

$$
\begin{aligned}
C_{i}(t)= & \eta_{1}\left(T+a_{i}-\frac{t_{i-1}+t_{i}}{2}\right)+\eta_{2}\left[t^{*}-\left(a_{i}+T\right)\right] \\
& +G \frac{x_{i}}{x_{0}}+p, \quad t \in\left(a_{k}, a^{*}\right] .
\end{aligned}
$$

The travel cost of the third class could be described by the following formula:

$$
\begin{aligned}
C_{i}(t)= & \eta_{1}\left(T+a_{i}-\frac{t_{i-1}+t_{i}}{2}\right)+\eta_{3}\left[\left(a_{i}+T\right)-t^{*}\right] \\
& +G \frac{x_{i}}{x_{0}}+p, \quad t \in\left(a^{*}, a_{l}\right] .
\end{aligned}
$$

The travel cost of the last class could be described by the following formula:

$$
\begin{aligned}
C_{i}(t)= & \eta_{1}\left(T+a_{i}-\frac{t_{i-1}+t_{i}}{2}\right)+\eta_{3}\left[\left(a_{i}+T\right)-t^{*}\right] \\
& +G \frac{x_{i}}{x_{0}}+p \theta, \quad t \in\left(a_{l}, a_{n}\right] .
\end{aligned}
$$

All the commuters have been divided into four classes. In general, the equilibrium condition among commuters can be statd as follows: every commuter is unable to find another departure time to reduce the total waiting time. This model can be described as the following programming problem:

$$
\min \sum_{i=1}^{n} x_{i}\left(a_{i}-\frac{t_{i-1}+t_{i}}{2}\right)
$$

s.t. (9)-(12)

$$
\begin{gathered}
0 \leq x_{i} \leq s \quad i=1, \ldots, n \\
a_{0}<t_{1}<t_{2}<\cdots<t_{n-1}<a_{n} \\
t_{i} \leq a_{i} \quad i=1, \ldots, n \\
\sum_{1}^{n} x_{i}=N \\
C_{1}(t)=C_{2}(t)=\cdots=C_{n}(t) .
\end{gathered}
$$

The objective function (13) means to minimize the total waiting time. Constraints (9)-(12) state the travel cost in each urban railroad. Constraint (14) guarantees the number of commuters in each urban railroad should be less than the capacity. Constraint (15) states that the commuters should obey the first-come-first-served basis. Constraint (16) states the time which the last commuter gets on urban railroad before the departure time of the urban railroad. Constraint (17) represents the flow conservation. Constraint (18) guarantees the travel costs of each commuter are equal at equilibrium.

\section{Numerical Results}

In this section, the models are simulated by LINGO 11. A set of numerical results is presented to illustrate the effects of considerable factors. It is assumed that there are 24 trains in 2 hours. Table 1 provides parameter values used for computational experiments. In this paper, $\eta_{1}, \eta_{2}$, and $\eta_{3}$ should obey $\eta_{2}<\eta_{1}<\eta_{3}[14]$.

Firstly, the schedule delay penalty model with small travel demand has been studied. The travel demand equals 13000. According to the assumptions in this study, commuters choose their departure time to minimize the total travel cost. When $\theta=0.45$, the objective function reaches the optimal. If the discount is larger or smaller than 0.45 , the objective function cannot get optimal. For example, the fare discount has no effect when $\theta=0$ in all time of the rush hours. Table 1 describes the change of the discount with the commuters' choice. 


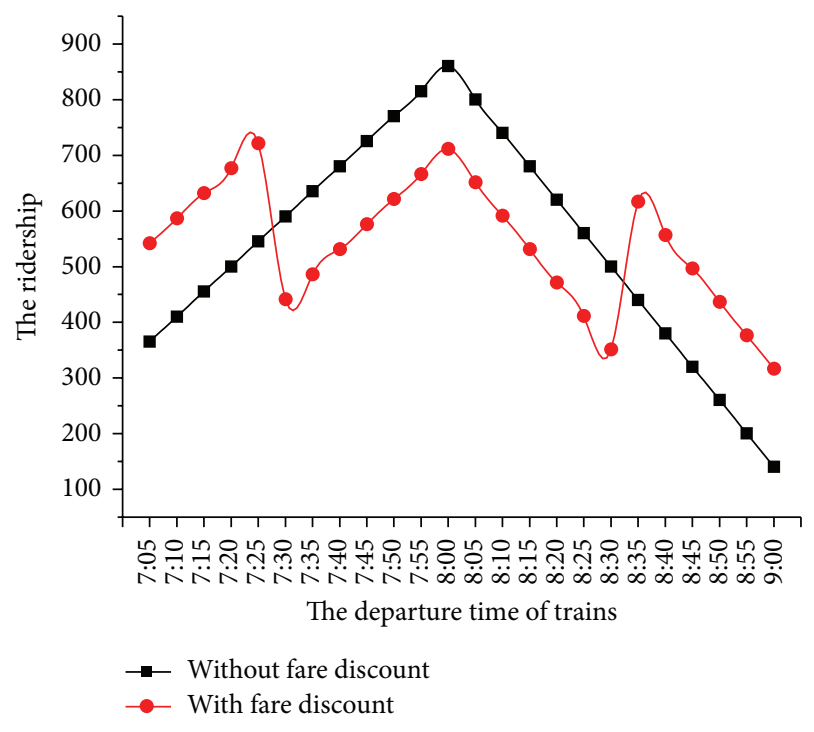

FIGURE 1: The result of fare discount model under equal pressure.

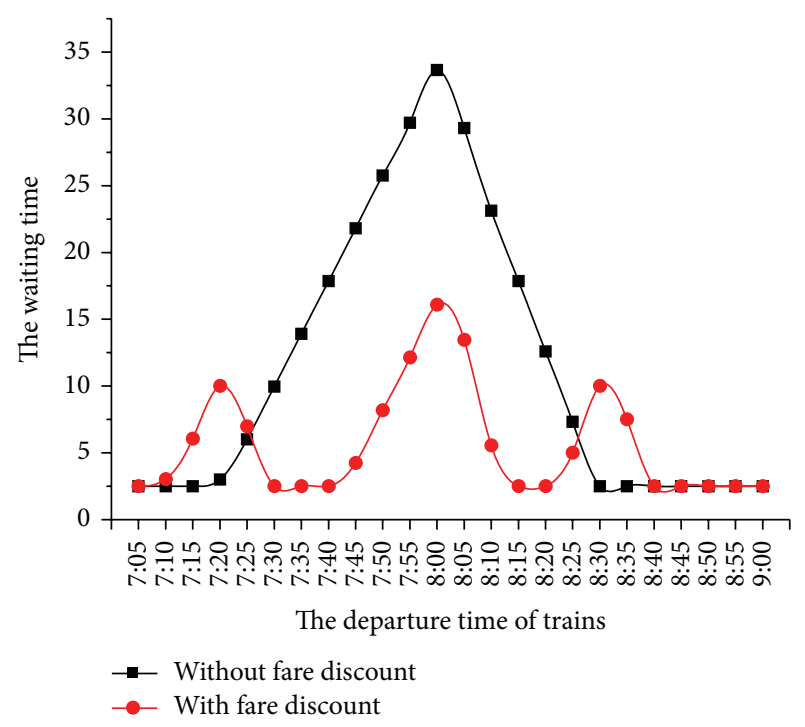

Figure 2: The result of fare discount model to minimize the total waiting time.

From Figure 1, we find the ridership in the no discount time interval has reduced 1939. The travel cost has been reduced from 5.72 RMB to 5.17 RMB, and the rate of the discount is 0.45 . That means when the discount is the 0.45 of the ticket fare, there are $21.59 \%$ of the commuters who change their departure time. Without discount, we find that there are free seats in each vehicle in the last 10 minutes. After the discount, there is no free seat in each vehicle. The congestion in the middle time interval has been alleviated. That is to say, the system resources have been effectively utilized.

Different from the schedule delay penalty model with small travel demand, this model has a large travel demand which equals 32000 . From Figure 2, we find the max waiting time has been reduced from 33.65 minutes to 16.08 minutes. The total waiting time has been reduced from 383443.2
TABLE 1: Parameter values used in computational experiments.

\begin{tabular}{lcc}
\hline Parameter & Symbol & Value \\
\hline The travel time & $T$ & 20 minutes \\
The waiting time & $\Delta t$ & 5 minutes \\
The unit cost of travel time & $\eta_{1}$ & $0.038 \mathrm{RMB}$ per min \\
The unit cost of schedule delay & $\eta_{2}$ & $0.03 \mathrm{RMB}$ per min \\
time-early & & \\
The unit cost of schedule delay & $\eta_{3}$ & $0.04 \mathrm{RMB}$ per min \\
time-late & $x_{0}$ & 240 \\
The number of seats & $p$ & $2 \mathrm{RMB}$ \\
Ticket price & $G$ & 0.8 \\
A parameter of body congestion & &
\end{tabular}

minutes to 184076.8 minutes, when the rate of the discount is 0.52 . If the discount rate is larger than 0.52 , the fare discounts for the trasportantion system have no effective.

For seeking the minimum travel cost, some commuters reach at station early. Long waiting times have been explained through this numerical result. This means the discount of urban railroad fare is an effective approach to solve congestion in the platform. The congestion in the middle time interval has been alleviated and the system resources have been effectively utilized.

According to travel demand size, the schedule delay penalty models can be divided into two kinds. This paper finds both models alleviate the congestion in the platform and vehicle. The special model is suitable in the small travel demand size. Different from the speical model, the schedule delay penalty model with large travel demand is more feasible in real life.

\section{Conclusions}

In this paper, the schedule delay model has been constructed in urban railroad where the urban railroad fare adjustment is considered. To determine fare adjustments, the paper constructs two user-equilibrium models duo to travel demand size. The first model is a special case of the second model when there is a small travel demand. The second model is the main model which considers big travel demand. Commuters are loaded on a first-come-first-served basis and must wait for the following vehicles. Two numerical examples are provided.

The first example is based on a small travel demand. The number of commuters has reduced and alleviated the congestion in the no discount time interval if there is ticket fare adjustment. Different from the second model, this model puts more emphasis on the rational use of resources and avoids the waste. That is to say, the system resources would be effectively utilized when the fare discount can be implemented.

The second example is based on a large travel demand, in which numerical analysis is performed. From the numerical results, some interesting observations are made with practical implementations. Firstly, the discount should only be used when the travel demand size is sufficiently large. In the small size, the former model has been enough. Secondly, the 
max waiting time has reduced nearly a half. This means the price discount is an effective approach to solve congestion in platform and carriage. Thirdly, under the discount of the railroad fare, commuters have saved the travel cost significantly.

Finally, both models have alleviated the congestion in the middle time interval with discount, and the system resources have been effectively utilized. This is our government desired result. The presented model and concept in this study can be further explored in either theoretical direction or practical applications. In reality, the population is heterogeneous due to income level, perception on general travel cost's composition, and so forth and can be divided into multiple classes. A multiclass model and serviceability analyses would be more flexible and feasible to be applied in different regions with diverse population configurations.

\section{Conflict of Interests}

The authors declare that there is no conflict of interests regarding the publication of this paper.

\section{Acknowledgments}

This paper is partly supported by National Basic Research Program of China (2012CB725400) and NSFC (71271023 and 71322102).

\section{References}

[1] H. Yang and X. Wang, "Managing network mobility with tradable credits," Transportation Research Part B, vol. 45, no. 3, pp. 580-594, 2011.

[2] S. I. Y. Chien and C. F. M. Tsai, "Optimization of fare structure and service frequency for maximum profitability of transit systems," Transportation Planning and Technology, vol. 30, no. 5, pp. 477-500, 2007.

[3] W. S. Vickrey, "Some implications of marginal cost pricing of public utilities," American Economis Review, vol. 45, no. 2, pp. 605-620, 1955.

[4] H. Mohring, "Optimization and scale economies in urban bus transportation," American Economic Review, vol. 62, no. 4, pp. 591-604, 1972.

[5] H. Mohring, Transportation Economics, Ballinger, Cambridge, Mass, USA, 1976.

[6] S. Vickrey, "Theory and transportation investment," American Economic Review, vol. 59, no. 2, pp. 414-431, 1969.

[7] R. Arnott, A. de Palma, and R. Lindsey, "A structural model of peak-period congestion: a traffic bottleneck with elastic demand," The American Economic Review, vol. 83, no. 1, pp. 161179, 1993.

[8] T. Yao, M. M. Wei, B. Zhang, and T. Friesz, "Congestion derivatives for a traffic bottleneck with heterogeneous commuters," Transportation Research B, vol. 46, no. 10, pp. 1454-1473, 2012.

[9] F. Xiao, W. Shen, and H. Michael Zhang, "The morning commute under flat toll and tactical waiting," Transportation Research B, vol. 46, no. 10, pp. 1346-1359, 2012.

[10] Q. Tian, H. J. Huang, and W. H. K. Lam, "Modeling the crowding effects of transit system on commuters' departure time choice behaviors," in Proceedings of the 9th International Conference on Industrial Management, pp. 386-393, 2008.

[11] E. J. Gonzales and C. F. Daganzo, "Morning commute with competing modes and distributed demand: user equilibrium, system optimum, and pricing," Transportation Research B: Methodological, vol. 46, no. 10, pp. 1519-1534, 2012.

[12] A. de Palma and S. Proost, "Imperfect competition and congestion in the city," Journal of Urban Economics, vol. 60, no. 2, pp. 185-209, 2006.

[13] R. Zhong, A. Sumalee, and T. Maruyama, "Dynamic marginal cost, access control, and pollution charge: a comparison of bottleneck and whole link models," Journal of Advanced Transportation, vol. 46, no. 3, pp. 191-221, 2012.

[14] K. A. Small, “The scheduling of consumer activities: work trips," The American Economic Review, vol. 72, no. 3, pp. 467-479, 1982.

[15] M. Kraus and Y. Yoshida, "The commuter's time-of-use decision and optimal pricing and service in urban mass transit," Journal of Urban Economics, vol. 51, no. 1, pp. 170-195, 2002.

[16] M. Kraus, "A new look at the two-mode problem," Journal of Urban Economics, vol. 54, no. 3, pp. 511-530, 2003.

[17] H. J. Huang, Q. Tian, H. Yang, and Z. Y. Gao, "Modeling the equilibrium bus riding behavior in morning rush hour," in Proceedings of the 9th Annual Conference of the Hong Kong Society of Transportation Studies, pp. 434-442, Hong Kong, China, 2004.

[18] H.-J. Huang, Q. Tian, H. Yang, and Z.-Y. Gao, "Modal split and commuting pattern on a bottleneck-constrained highway," Transportation Research E: Logistics and Transportation Review, vol. 43, no. 5, pp. 578-590, 2007.

[19] Z. Li and D. A. Hensher, "Crowding and public transport: a review of willingness to pay evidence and its relevance in project appraisal," Transport Policy, vol. 18, no. 6, pp. 880-887, 2011.

[20] A. Schoebel and S. Scholl, "Line planning with minimal traveling time," in Proceedings of 5th Workshop on Algorithmic Methods and Models for Optimization of Railroads, ATMOS, Palma de Mallorca, Spain, 2005.

[21] B. Yu, Z. Yang, and J. Yao, "Genetic algorithm for bus frequency optimization," Journal of Transportation Engineering, vol. 136, no. 6, pp. 576-583, 2010. 


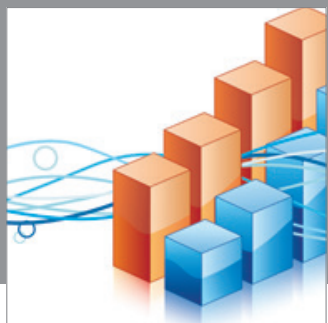

Advances in

Operations Research

mansans

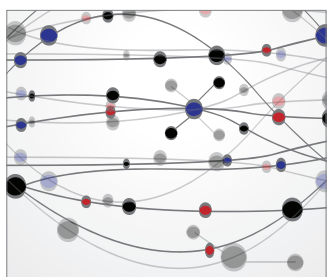

The Scientific World Journal
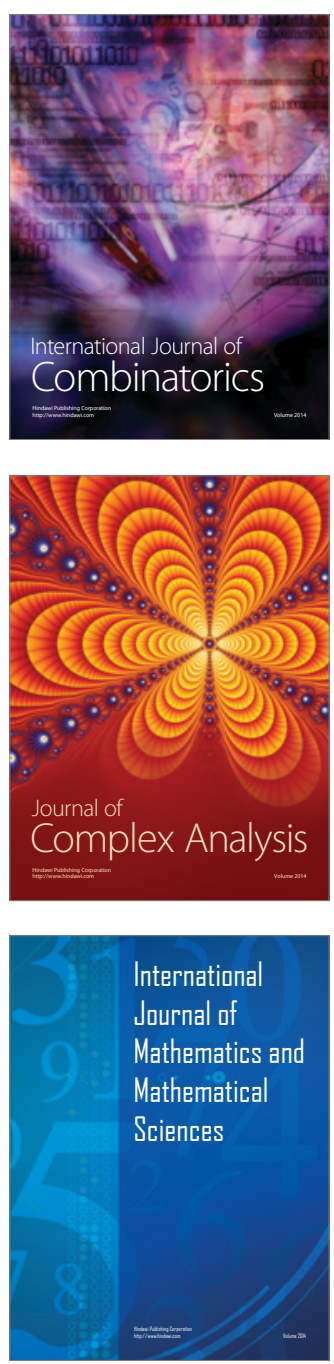
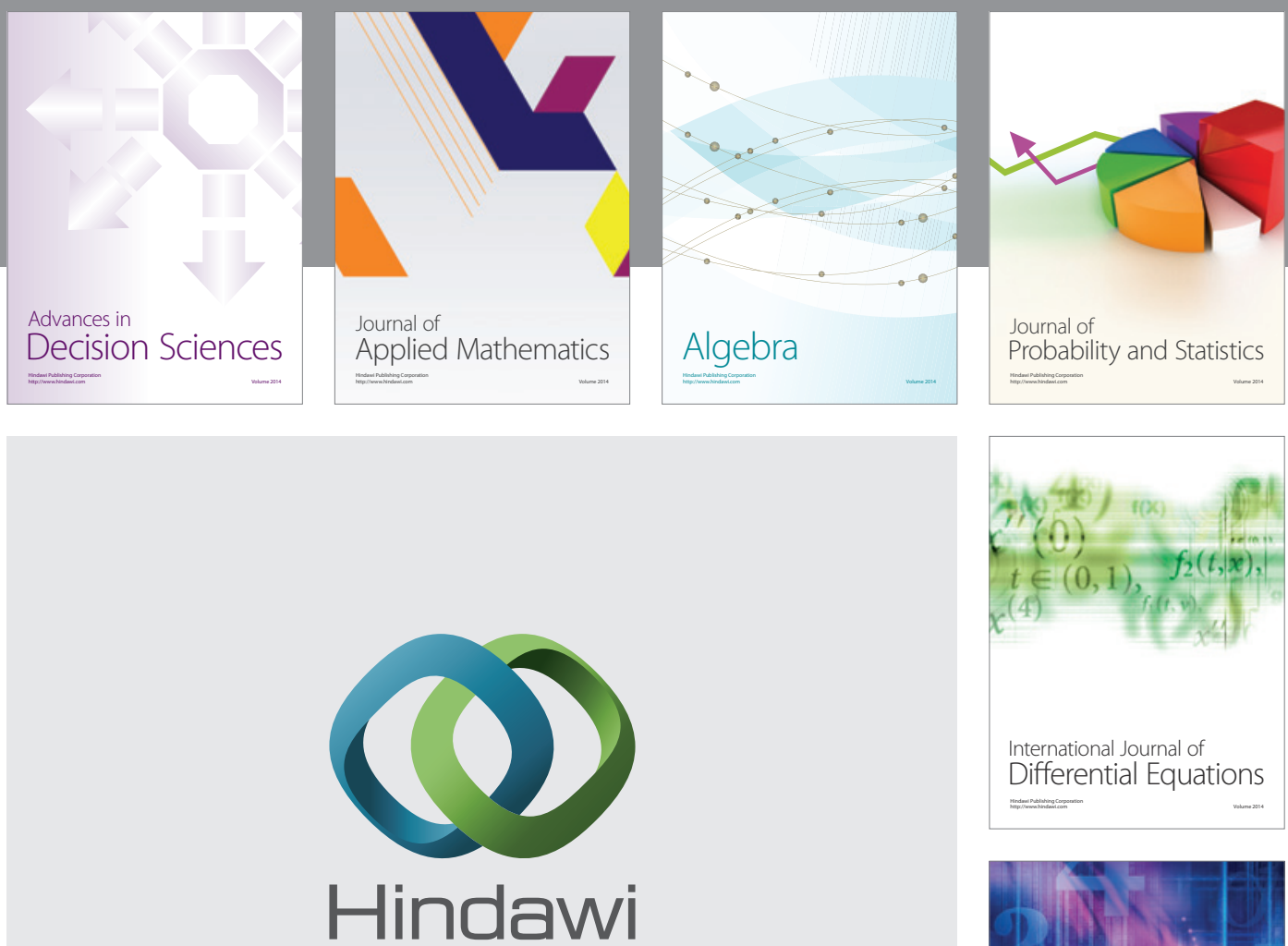

Submit your manuscripts at http://www.hindawi.com
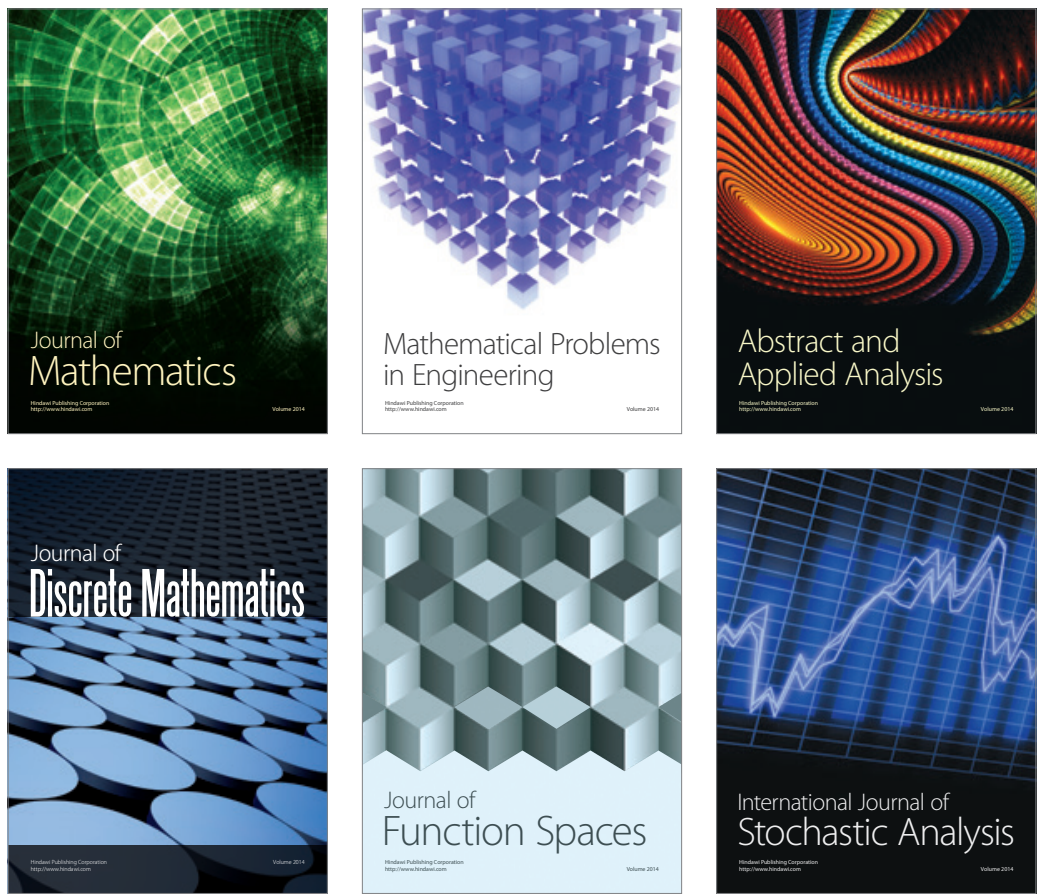

Journal of

Function Spaces

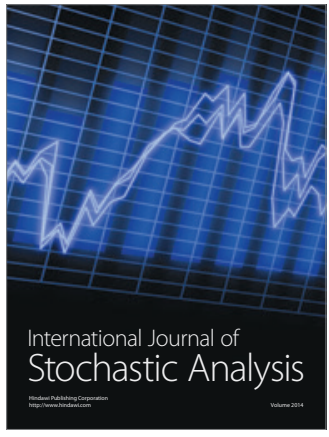

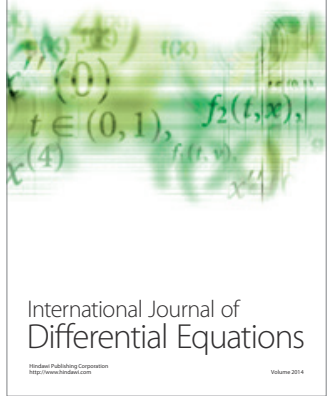
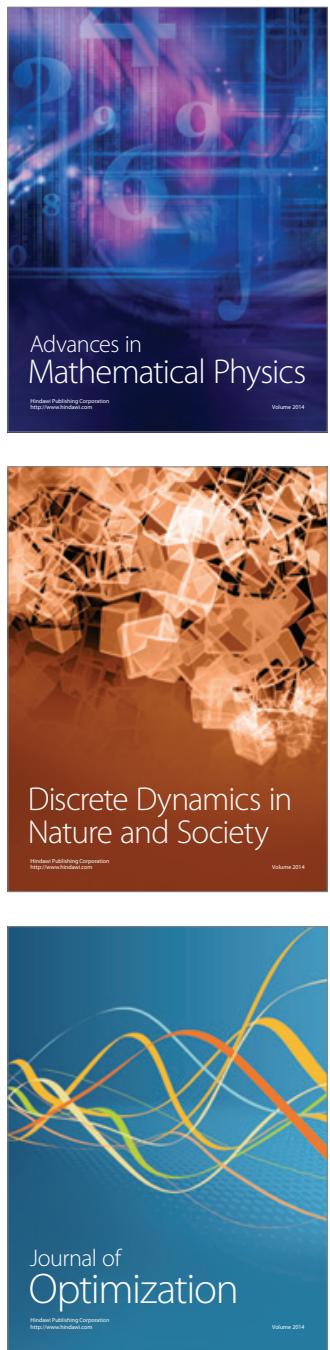